\title{
Evaluación de un inhibidor utilizado en soluciones ácidas bajo condiciones hidrodinámicas controladas ${ }^{(\bullet)}$
}

\author{
V.M. Salinas-Bravo ${ }^{(*)}$ y J.G. González-Rodríguez ${ }^{(*)}$ \\ Resumen Utilizando el electrodo cilíndrico rotatorio y técnicas electroquímicas, se estudió la eficiencia de la \\ protección contra la corrosión de un inhibidor comercial utilizado en soluciones de ácido clorhídrico \\ con y sin adiciones de tiourea y bifluoruro de amonio. El fenómeno de la corrosión se investigó en \\ condiciones hidrodinámicas controladas, seleccionadas para simular las que se establecen en una \\ tubería a distintas velocidades de flujo. Se encontró que la corrosión del acero al carbono en \\ soluciones de ácido clorhídrico con inhibidor de corrosión está controlada principalmente por \\ activación, mientras que en soluciones ácidas con inhibidor y adiciones de tiourea y bifluoruro de \\ amonio está controlada principalmente por transferencia de masa. Por otra parte, la velocidad del \\ flujo de las soluciones ácidas afecta de ‘manera negativa a la eficiencia de protección del inhibidor \\ estudiado en el intervalo de concentración de 0,2 a $1,0 \%$.
}

Palabras clave: Electrodo cilíndrico rotatorio. Acero al carbono. Corrosión. Ácido clorhídrico. Inhibidor. Velocidad de flujo.

\section{Evaluation of acid inhibitors under controlled hydrodynamic conditions}

\begin{abstract}
The cylindrical rotating electrode and electrochemical techniques were used to investigate the protection against corrosion of a commercial inhibitor used in hydrochloric acid solutions with and without additions of thiourea and ammonium bifluoride. The corrosion phenomenon was investigated under controlled hydrodynamic conditions. These were chosen to simulate those found in a pipe at different flow velocities. It was found that corrosion of mild steel in hydrochloric acid solutions with inhibitor is mainly controlled by activation, whereas in acid solutions with inhibitor added with thiourea and ammonium bifluoride it is mainly controlled by mass transfer. On the other hand, flow velocity of the acidic solutions affects negatively the efficiency of the inhibitor in the concentration range of 0.2 to $1.0 \%$.
\end{abstract}

Keywords: Rotating cylinder electrode. Mild steel. Corrosion. Hydrochloric acid. Inhibitor. Flow velocity.

\section{INTRODUCCIÓN}

Una aplicación industrial de los inhibidores de corrosión con sustancias ácidas se encuentra en la limpieza química de los tubos intercambiadores de calor de los generadores de vapor de las centrales térmicas.

(•) Trabajo recibido el día 25 de marzo de 1996.

(*) Instituto de Investigaciones Eléctricas. Dpto. FísicoQuímica Aplicada. Aptdo. postal 1-475. Cuernavaca, Morelos, Mor. 62001 (México).
Durante el procedimiento de limpieza con soluciones químicas, la velocidad del fluido es muy importante en la eficiencia de la eliminación de los depósitos de las tuberías. Es deseable una velocidad mínima del fluido para asegurar que los depósitos eliminados se incorporen en la solución. Sin embargo, la velocidad máxima del fluido está definida por la necesidad de mantener una "película" de inhibidor sobre la superficie del metal que impida la corrosión acelerada del mismo durante la limpieza química.

Ante esta situación, y con el propósito de llevar a cabo una operación de limpieza química segura, 
es decir, sin ataque corrosivo excesivo al metal de las tuberías, es necesario conocer cómo afecta la velocidad del flujo de la solución ácida a la efectividad del inhibidor de corrosión utilizado.

En la literatura existen trabajos relacionados con el uso del electrodo cilíndrico rotatorio para el estudio del comportamiento de materiales metálicos en medios corrosivos (1-4). En estos estudios se han establecido correlaciones que implican las condiciones hidrodinámicas del sistema y el efecto de éstas en el comportamiento frente a la corrosión de los materiales. De esta manera, es posible conocer aquellos parámetros que afectan al comportamiento frente a la corrosión de un material en un sistema en particular, como puede ser el electrodo cilíndrico rotatorio (por ej., esfuerzo de corte, transferencia de masa, etc.), y a partir de estos parámetros determinar el comportamiento de otro sistema hidrodinámico diferente, como el que se establece en una tubería. El trabajo con este procedimiento es menos complicado y la información se puede obtener en menos tiempo si se compara con la manera tradicional de probar inhibidores a través de la construcción de circuitos de prueba en los que se utilizan bombas, intercambiadores de calor, válvulas, etc.

La hipótesis que se emplea para asegurar la misma interacción entre el material y el fluido, tanto a nivel industrial (tubería) como de laboratorio (electrodo cilíndrico rotatorio), es que el dispositivo utilizado en el laboratorio para simular la forma geométrica a nivel industrial deberá operar de tal manera que el intervalo del esfuerzo de corte producido por el fluido sea el mismo que se produce en la forma geométrica a escala industrial.

El esfuerzo cortante, $\tau$, producido por un fluido sobre la superficie de un cilindro al rotar en el fluido está definido como:

$$
\tau_{\text {(cil) }}=f / 2 \rho V^{2}
$$

donde $f$ es el factor de fricción, $\rho$ es la densidad del fluido y $V$ es la velocidad periférica del cilindro. En realidad, el esfuerzo de corte, $\tau$, es una medida de la rapidez de la transferencia de momento hacia la pared del cilindro.

Por otra parte, el esfuerzo cortante, $\tau$, producido por un fluido sobre la superficie de un tubo al fluir por su interior, está definido como:

$$
\tau_{\text {(tubo) }}=f / 8 \rho V^{2}
$$

donde $f$ es el factor de fricción, $\rho$ es la densidad del fluido y $V$ es la velocidad del fluido en el interior del tubo.

Al igualar ambos esfuerzos cortantes y sustituyendo los valores adecuados del factor de fricción y del número de Reynolds en ambos sistemas, es posible calcular la velocidad de rotación del cilindro que sea equivalente a una determinada velocidad del fluido en una tubería (1).

\section{ENSAYOS}

\subsection{Preparación de las probetas}

Se prepararon probetas cilíndricas a partir de una barra de acero al carbono. Las dimensiones de dichas probetas eran $12 \mathrm{~mm}$ de diámetro externo $\times$ $8 \mathrm{~mm}$ de longitud. Se realizó una perforación de 6 $\mathrm{mm}$ de diámetro en el centro de las probetas para que éstas se pudieran montar en el eje del rotor del cilindro rotatorio. La superficie expuesta de las probetas se desbastó con papel abrasivo de diferentes grados, empezando con un grado grueso hasta terminar con grado 600 .

\subsection{Materiales y soluciones de ensayo}

El material utilizado en la fabricación de las probetas fue acero al carbono, cuya composición química, expresada en tanto por ciento en masa, era la siguiente: 0,23 C, 0,42 Mn, 0,15 Si y 0,04 S. Los ensayos se llevaron a cabo en una solución de $\mathrm{HCl}$ $5 \%$ en masa con adiciones de 0,2 a $1,0 \%$ de inhibidor de la corrosión, y en solución del $5 \%$ en masa $\mathrm{HCl}+3 \%$ bifluoruro de amonio $+1 \%$ tiourea con adiciones de 0,2 a $1,0 \%$ de inhibidor, ambas a $65^{\circ} \mathrm{C}$. Se utilizó un inhibidor comercial de base orgánica, formador de película, conocido comercialmente con el nombre de Rodine- 213.

Para el estudio del efecto de la velocidad de flujo de la solución, se seleccionaron las siguientes velocidades de rotación (rpm) del cilindro: $0,1.157$, 2.360 , y 3.580 . Estas velocidades de rotación equivalen a una velocidad de flujo de $0,1,0,2,0$ y 3,0 $\mathrm{m} / \mathrm{s}$, respectivamente, en una tubería de $45 \mathrm{~mm}$ de diámetro interno.

Los ensayos se llevaron a cabo en un electrodo rotatorio cilíndrico de la marca EG \& G, de Princeton Applied Research, que dispone de control automático de la velocidad de rotación del eje rotor en el que se inserta el cilindro.

Una vez instalado el electrodo de acero al carbono en el eje rotor, se sumergió en la solución de prueba que se había vertido en una celda de corrosión de vidrio Pyrex que estaba equipada con dos electrodos auxiliares de grafito, un termómetro y un capilar de Luggin que permite el contacto entre el electrólito y el electrodo de referencia utilizado, que fue el de calomelanos saturado (ECS). La celda de vidrio Pyrex, a su vez, estaba rodeada por un manto de calentamiento a través del cual se lleva la solución a la temperatura deseada. El control de la temperatura se realizó por medio de un controlador Variac conectado al manto de calentamiento. 
Con objeto de tener condiciones superficiales iguales en los electrodos de acero al carbono, y debido a que estos se oxidan rápidamente si se preparan con antelación a los ensayos, las probetas, previamente a cada ensayo, se desbastaron con papel abrasivo de grado 600, se lavaron con agua destilada y se desengrasaron con metanol y acetona.

Las mediciones electroquímicas se llevaron a cabo utilizando una interfase electroquímica de la marca ACM Instruments, que se controla mediante un ordenador personal que almacena y procesa los datos de cada uno de los ensayos.

La técnica electroquímica utilizada para determinar la velocidad de corrosión del acero al carbono en las diferentes soluciones fue la de polarización potenciodinámica, conocida como extrapolación de Tafel.

Previamente a la realización del ensayo mediante la técnica electroquímica, se dio tiempo a que el potencial de corrosión de la muestra permaneciera razonablemente constante y se procedió a polarizar el electrodo. El intervalo de potencial de polarización para la técnica de Tafel fue de $100 \mathrm{mV}$, tanto en el sentido anódico como catódico, con respecto al potencial libre de corrosión, y la velocidad de barrido del potencial fue de $0,3 \mathrm{mV} / \mathrm{s}$.

\section{RESULTADOS Y DISCUSIÓN}

La figura 1 muestra el efecto de la concentración del inhibidor en la velocidad de corrosión del acero al carbono a distintas velocidades de rotación del

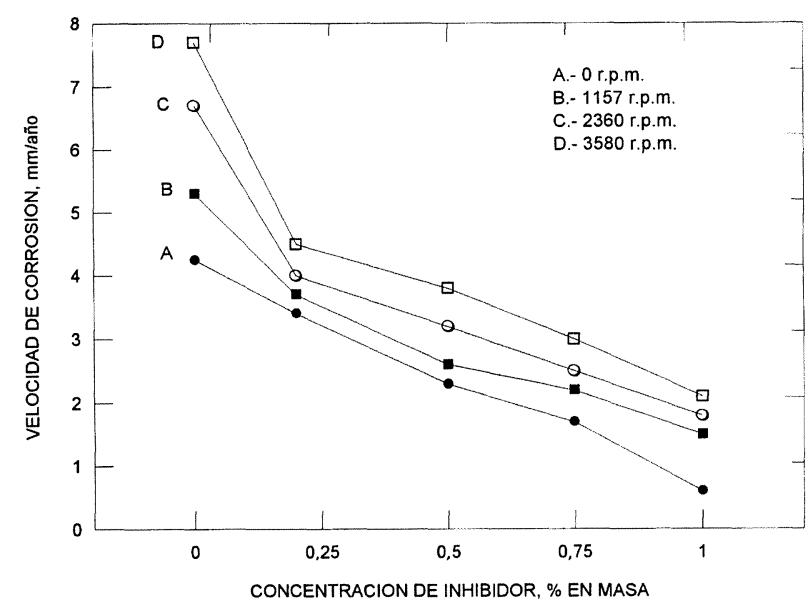

FIG. 1.- Efecto de la concentración de inhibidor en la velocidad de corrosión del acero al carbono en solución de $5 \% \mathrm{HCl}$ a $65^{\circ} \mathrm{C}$, a distintas velocidades de rotación del cilindro.

FIG. 1.- Effect of inhibitor concentration on the corrosion rate of mild steel in $5 \% \mathrm{HCl}$ at different rotating cylinder speeds. cilindro en solución $\mathrm{HCl} 5 \%$ a $65{ }^{\circ} \mathrm{C}$. Se observa claramente que la velocidad de corrosión del acero al carbono disminuye a medida que aumenta la concentración de inhibidor para cada velocidad de rotación utilizada. Sin embargo, es notorio cómo un incremento de la velocidad de rotación del cilindro, a una concentración de inhibidor constante, propicia un incremento de la velocidad de corrosión del acero. El aumento de la velocidad de corrosión se explica debido a que al aumentar la velocidad de rotación del cilindro, el esfuerzo de corte entre el fluido y la pared del cilindro también aumenta, lo que impide la adsorción del inhibidor en el metal y favorece la reacción de corrosión.

Cuando se agrega tiourea y bifluoruro de amonio a la solución inhibida de ácido clorhídrico, se obtienen velocidades de corrosión superiores (Fig. 2). En esta figura se observa que adiciones de inhibidor mayores del $0,2 \%$, a velocidades de rotación del cilindro inferiores a $1.157 \mathrm{rpm}$, prácticamente no tienen efecto en la disminución de la velocidad de corrosión del acero. Este hecho es indicativo de que en este tipo de soluciones, velocidades de rotación del cilindro relativamente bajas no impiden la adsorción del inhibidor en la superficie del metal. A velocidades de rotación superiores a $1.157 \mathrm{rpm}$, la adsorción del inhibidor se ve impedida por el efecto de mayores esfuerzos de corte en la superficie del electrodo.

El resultado anterior pone de manifiesto la importancia de agregar otras sustancias químicas a la solución de ácido para formular la solución

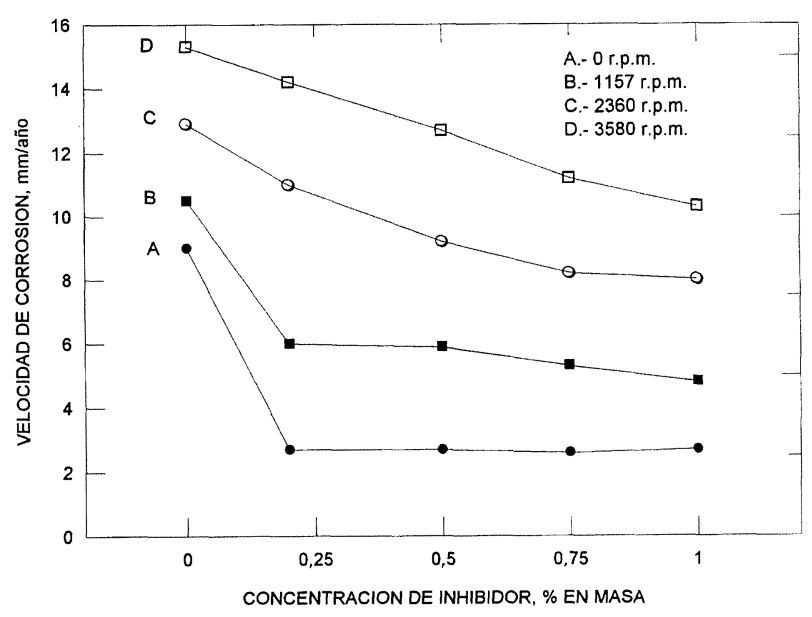

Fig. 2.- Efecto de la concentración de inhibidor en la velocidad de corrosión del acero al carbono en una solución de $5 \% \mathrm{HCl}+3 \%$ bifloruro de amonio $+1 \%$ tiourea a $65{ }^{\circ} \mathrm{C}$, a distintas velocidades de rotación del cilindro.

FIG. 2.- Effect of inhibitor concentration on the corrosion rate of mild steel in $5 \% \mathrm{HCl}+3 \%$ ammonium bifluoride $+1 \%$ thiourea at $65{ }^{\circ} \mathrm{C}$ at different rotating cylinder speeds. 
limpiadora; dichas sustancias pueden interferir con la capacidad de un inhibidor para proteger un metal de la corrosión. Esto también realza la importancia de que la evaluación de un inhibidor de la corrosión no se debe llevar a cabo sólo con el ácido utilizado, sino que los ensayos deberán incluir además del inhibidor todas las sustancias que se utilicen en las formulaciones de limpieza química.

Un análisis de la correlación de transferencia de masa para el electrodo cilíndrico rotatorio (4) permite conocer los fenómenos que controlan la cinética de un sistema determinado.

Cuando se representa gráficamente el $\log$ de $R_{\mathrm{e}}$ frente al log de la velocidad de corrosión, se obtiene una recta cuya pendiente determina el control de la cinética del sistema.

El número de Reynolds $\left(R_{\mathrm{e}}\right)$ para el electrodo cilíndrico rotatorio está definido como:

$$
R_{\mathrm{e}}=D V / v
$$

donde $D$ es el diámetro del cilindro, $V$ es la velocidad periférica y $v$ es la viscosidad cinemática del fluido.

Cuando la pendiente resultante es inferior a 0,7 se considera que el proceso está controlado por activación, y cuando es igual o mayor de 0,7 el proceso está controlado por transferencia de masa o difusión.

Para el caso de las soluciones ácidas que contienen únicamente inhibidor, el proceso que domina el control de la cinética de corrosión es principalmente la activación (Fig. 3). Sin embargo, en la figura 4 se

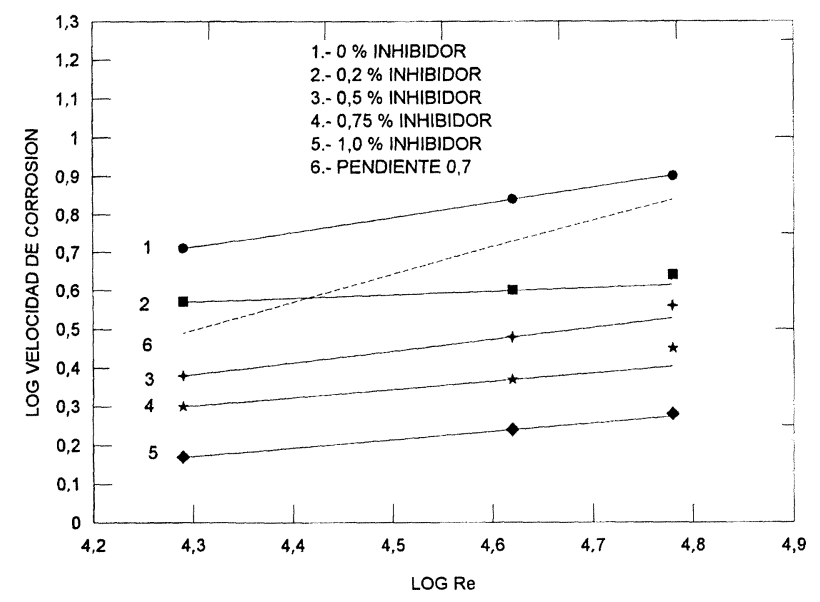

FIG. 3.- Efecto de la velocidad de flujo en la velocidad de corrosión del acero al carbono en soluciones de $5 \% \mathrm{HCl}$ a $65{ }^{\circ} \mathrm{C}$, con diferentes concentraciones de inhibidor.

FIG. 3.- Effect of flow velocity on the corrosion rate of mild steel in $5 \% \mathrm{HCl}$ at $65{ }^{\circ} \mathrm{C}$ with different inhibitor concentrations.

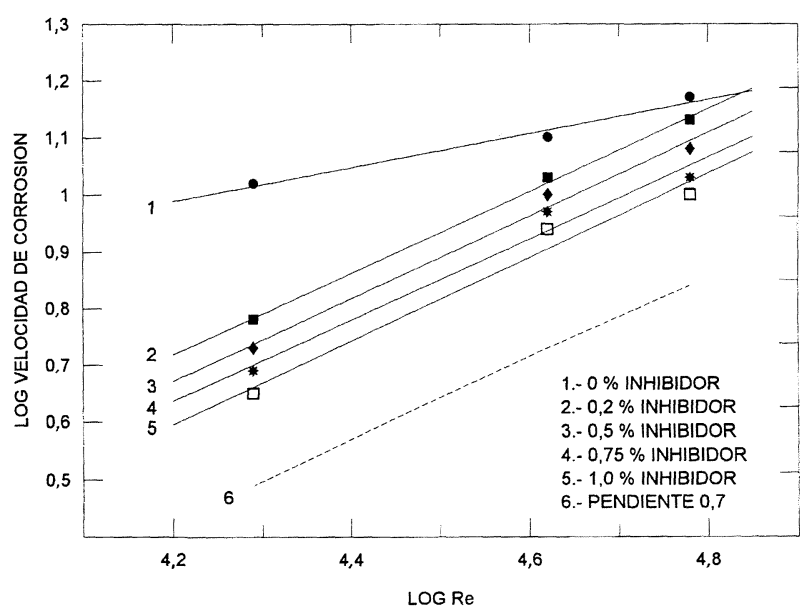

FIG. 4.- Efecto de la velocidad de flujo en la velocidad de corrosión del acero al carbono en soluciones de $5 \% \mathrm{HCl}+3 \%$ bifluoruro de amonio $+1 \%$ tiourea a $65{ }^{\circ} \mathrm{C}$, con diferentes concentraciones de inhibidor.

FIG. 4.- Effect of flow velocity on the corrosion rate of mild steel in $5 \% \mathrm{HCl}+3 \%$ ammonium bifluoride $+1 \%$ thiourea at $65{ }^{\circ} \mathrm{C}$ with different inhibitor concentrations.

puede observar que al adicionar bifluoruro de amonio y tiourea a la solución ácida con inhibidor, el proceso que controla la cinética de corrosión del acero al carbono cambia de un control por activación a otro controlado principalmente por la difusión de especies químicas hacia la superficie del electrodo.

La figura 5 muestra las curvas de polarización potenciodinámica obtenidas a distintas velocidades de rotación del cilindro en una solución de $5 \% \mathrm{HCl}$, $0,75 \%$ de inhibidor Rodine $213,1 \%$ de tiourea y $3 \%$ de bifloruro de amonio a $65{ }^{\circ} \mathrm{C}$. En estas curvas se puede observar cómo un aumento en la velocidad de rotación del cilindro propicia un incremento en la cinética de corrosión del material.

\section{CONCLUSIONES}

- El incremento de la velocidad del fluido de las soluciones ácidas probadas propicia un incremento de la velocidad de corrosión del acero al carbono.

- La adición de bifluoruro de amonio y tiourea a la solución de ácido clorhídrico con inhibidor interfiere en la eficiencia de protección del inhibidor, obteniéndose valores de corrosión mayores y eficiencias de protección menores en comparación con soluciones únicamente con inhibidor.

- La adición de bifluoruro de amonio y tiourea cambia la cinética de corrosión de un control por 


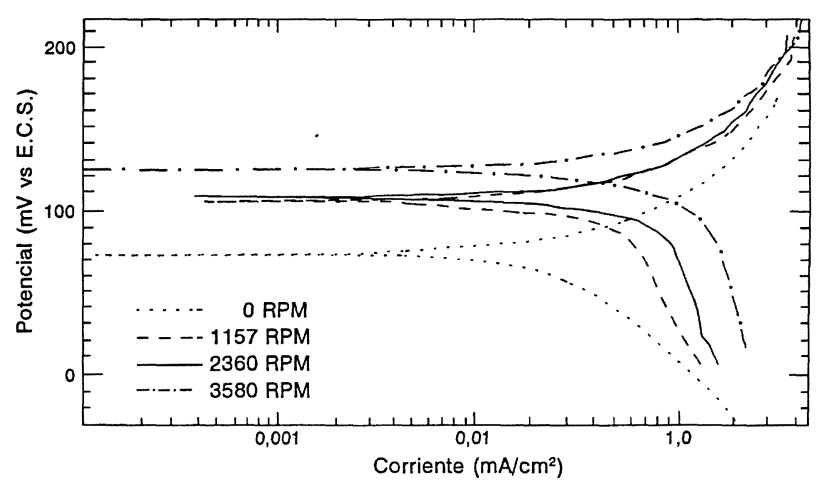

FIG. 5.- Curvas de polarización potenciodinámica obtenidas en una solución de $5 \% \mathrm{HCl}+3 \%$ bifluoruro de amonio $+1 \%$ tiourea $+0,75 \%$ de inhibidor Rodine 235 a $65{ }^{\circ} \mathrm{C}$. Efecto de la velocidad de rotación del cilindro.

FIG. 5.- Potentiodynamic polarization curves obtained in a solution of $5 \% \mathrm{HCl}+3 \%$ ammonium bifluoride $+1 \%$ thiourea $+0,75 \%$ inhibitor Rodine 235 at $65^{\circ}$ C. Effect of rotation speed of cylinder

activación en soluciones ácidas con inhibidor a un control por transferencia de masa.
- La utilización de técnicas electroquímicas para investigar la corrosión del sistema en estudio y el empleo del electrodo cilíndrico rotatorio para simular el efecto de la velocidad de fluido, constituyen herramientas importantes para la evaluación y selección de inhibidores de la corrosión.

\section{REFERENCIAS}

(1) Silverman, D.C. Rotating Cylinder Electrode. An Approach For Predicting Velocity Sensitive Corrosion. Corrosion/90 (NACE). Las Vegas, Nevada (EE.UU.), 1990: Paper No. 13.

(2) DeAn, S.W. Overview of Velocity Accelerated Corrosion Testing and Predictions. Corrosion/90 (NACE). Las Vegas, Nevada (EE.UU.), 1990: Paper No. 12.

(3) Chen, T., Moccari, A. y Macdonald, D.D. The Development of Controlled Hydrodinamic Techniques for Corrosion Testing. Corrosion/91 (NACE). Cincinnati, Ohio (EE.UU.), 1991: Paper No. 292.

(4) Eisemberg, M., Tobias, C.W. y Wilke, C.R. J. Electrochem. Soc., 101, 1954: 306. 